\title{
ESTRATEGIAS PARA EMPODERAR A LAS MUJERES EN RELACIÓN CON LOS DERECHOS REPRODUCTIVOS Y LA PLANIFICACIÓN FAMILIAR. REVISIÓN SISTEMÁTICA DESDE LAS CORRIENTES BIOÉTICAS
}

\begin{abstract}
Manuel José Peón-Ponce', Nina Méndez-Domínguez², Jorge Carlos Guillermo Herrera ${ }^{3}$
Resumen: El presente artículo informa de una investigación cuyo objetivo fue definir las estrategias para empoderar a las mujeres mexicanas en sus decisiones de planificación familiar y de establecer compromisos y responsabilidades con sus parejas. Se realizó una revisión sistemática, incluyendo PubMed, Redalyc y Scopus, y se incluyeron diez artículos originales de dos continentes y diversas poblaciones. De los estudios realizados en México y Latinoamérica ninguno proporcionó una propuesta para implementar mejoras en la adopción de un método de planificación familiar entre las mujeres que viven violencia de pareja. Se concluye que la capacitación y formación bioética del personal de salud puede abonar a lograr que el proceso de información y decisión por parte de la mujer o de la pareja sean llevados a cabo con apego a la bioética, buscando el beneficio individual y protección del bienestar de la mujer y, por ende, de su familia.
\end{abstract}

Palabras clave: derechos reproductivos, derechos humanos, planificación familiar, violencia de pareja, violencia doméstica, conducta anticonceptiva

Strategies to empower women in relation to reproductive rights and family planning. A systematic review from bioethical currents

\begin{abstract}
This article reports on research aimed at defining strategies to empower Mexican women in their family planning decisions and to establish commitments and responsibilities with their partners. A systematic review was conducted, including PubMed, Redalyc and Scopus, and ten original articles from two continents and diverse populations were included. Of the studies conducted in Mexico and Latin America, none provided a proposal to implement improvements in the adoption of a family planning method among women who experience intimate partner violence. It is concluded that bioethical training and education of health personnel can contribute to ensure that the information and decision-making process by the woman or the couple is carried out in accordance with bioethics, seeking individual benefit and protection of the wellbeing of the woman and, therefore, of her family.
\end{abstract}

Keywords: reproductive rights, human rights, family planning, intimate partner violence, domestic violence, contraceptive behavior

Estratégias para empoderar as mulheres em relaçáo aos direitos reprodutivo e de planejamento familiar: revisão sistemática desde las correntes bioéticas

Resumo: O presente artigo informa sobre uma pesquisa cujo objetivo foi definir as estratégias para empoderar as mulheres mexicanas em suas decisóes de planejamento familiar e de estabelecer compromissos e responsabilidades com seus companheiros. Foi realizada uma revisão sistemática, incluindo PubMed, Redalyc e Scopus, e se incluíram dez artigos originais de dois continentes e diversas populaçóes. Dos estudos realizados no México e na América Latina nenhum proporcionou uma proposta para implementar melhorias na adoçâo de um método de planejamento familiar entre as mulheres que sofrem violência conjugal. Conclui-se que a capacitação e formaçáo bioética do pessoal de saúde pode lograr alcançar que o processo de informação e decisão por parte da mulher ou do casal sejam levados a cabo com apego à bioética, buscando o beneficio individual e a proteçấo do bem estar da mulher e, por conseguinte, de sua família.

Palavras chave: direitos reprodutivos, direitos humanos, planejamento familiar, violência conjugal, violência doméstica, conduta anticonceptiva

\footnotetext{
${ }^{1}$ Departamento de Asuntos Jurídicos. Hospital Regional de Alta Especialidad de la Península de Yucatán, México.

${ }^{2}$ Hospital Regional de Alta Especialidad de la Península de Yucatán, México.

Correspondencia: nina.mendez@salud.gob.mx

${ }^{3}$ Enseñanza e Investigación. Hospital Regional de Alta Especialidad de la Península de Yucatán, México
} 


\section{Introducción}

En México, la normativa indica que los servicios de planificación familiar deben ser promovidos por los médicos, involucrando la información y educación sobre los métodos anticonceptivos e, incluso, que esto forma parte de las actividades básicas de promoción de la salud en los programas de servicio social médico comunitario. De este modo, las instancias gubernamentales y de salud cumplen con la meta de los servicios de planificación familiar(1-6).

En México se ha regulado el proceso de consentimiento informado específico para la aceptación (o rechazo) de los métodos de planificación familiar, significando esta medida una fortaleza y un logro bioético de trascendencia, al abonar directamente a la autonomía de las usuarias. Los datos del Instituto Nacional de Estadística y Geografía (INEGI) son contundentes en cuanto al éxito en el uso de los métodos de planificación familiar sobre la reducción de la natalidad, dado que, para 2007, se reportaron $2^{\prime} 655,083$ nacimientos y en el año 2017 se registraron 2'234,039 nacimientos(2,3). Según Mandujano, la píldora anticonceptiva de emergencia fue el anticonceptivo más vendido en México en 2017. Previamente, en 2012, Villegas reportó que en un periodo de 12 meses hubo mujeres que habían consumido hasta 36 veces la pastilla anticonceptiva de emergencia $(7,8)$.

Sin embargo, también se ha identificado que el proceso de consentimiento informado en nuestro país gira más en torno a conseguir la firma del formato que al proceso de información total y completa para lograr un consentimiento genuino.

Llama la atención el continuado crecimiento exponencial de la venta la dispensación y oferta de las píldoras anticonceptivas de emergencia, que se emplean cuando existe una exposición sin protección a relaciones sexuales, sin empleo de anticonceptivos o por no llevar un método de planificación familiar. Tal es la oferta, que las pastillas pueden comprarse en cualquier supermercado sin receta médica, con precios desde sesenta pesos.

La percepción que puede tenerse al respecto es que, dado que las píldoras anticonceptivas de emergencia no son consideradas como un méto- do habitual, pues solo se indican (como dice su nombre) en caso de emergencia (por fallo) o de violación, entonces las metas de planificación familiar pueden no estar alcanzándose de la mejor manera, pues, si así fuese, la popularidad, acceso y oferta de las píldoras anticonceptivas de emergencia no sería tan alarmante.

Las causas que están provocando el problema bioético sobre el cual versa nuestra propuesta están entrelazadas entre sí, y difícilmente pudiera una sola de ellas por separado generar directamente el problema. Explicadas como en un modelo multinivel incluyen, de lo general a lo particular:

(a) un sistema de gobierno que pondera las cifras y evalúa el éxito de los programas de planificación familiar como una medida meramente cuantitativa de reducción de natalidad y fecundidad;

(b) una sociedad que ha normalizado e incorporado la violencia a la mujer en diferentes ámbitos, desde los micromachismos hasta la coerción de las decisiones libres;

(c) servicios de salud que miden sus alcances en planificación familiar por "acciones" promotoras y no por intervenciones evaluadas cualitativa y cuantitativamente, así como su personal sanitario, que parece más interesado en la firma del consentimiento informado obstétrico que en proporcionar información y facilitar la comprensión y apego a los métodos de planificación;

(d) familias en las que se desarrollan las nuevas generaciones, en las cuales los derechos de la mujer son pasados por alto para privilegiar al hombre;

(e) relaciones de pareja permisivas, que incorporan y normalizan que la voluntad masculina sea privilegiada a toda costa, y

(f) mujeres que, inseguras, indecisas y condescendientes, temen abordar y proponer la decisión de los métodos de planificación familiar con su pareja.

En México, la violencia de pareja es un problema latente que, al combinarse con una cultura "machista" y las arraigadas creencias e ideas de cómo debe decidirse la planificación familiar, puede facilitar la coerción de los derechos reproductivos 
de las mujeres que viven violencia de pareja; sin embargo, es posible que el equipo multidisciplinario de salud pueda mitigar este problema. Esta vulneración de la libertad de elección puede no presentarse como una negativa expresa ni perceptible por el personal de salud que provee los servicios de planificación familiar, sino que suele manifestarse sutilmente.

Como bioeticistas, podemos y debemos ir más allá de alcanzar las metas del INEGI en México, o de que se esté firmando el consentimiento informado. Dado que nuestro quehacer implica no solo los fines sino también sus medios, podemos encontrar en los "cómo" (más que en los "cuánto”) las vías para lograr mucho más. Podemos mejorar las estrategias a la luz de la evidencia, para que la decisión de aquellas mujeres que viven violencia de pareja sea auténtica, autónoma, libre e informada. Debemos procurar en todo momento la defensa de la vida humana, no cerrando los ojos ante el incremento del uso de las píldora anticonceptiva de emergencia o la baja aceptación de métodos de planificación familiar después de un aborto o pérdida fetal/neonatal (que pone en riesgo a la madre), pues en ambos casos pudiera además existir, de acuerdo con la evidencia revisa$\mathrm{da}$, un trasfondo de violencia de pareja que afecta la decisión sobre los métodos de planificación familiar que elige (o rechaza) la mujer.

De acuerdo con la evidencia existente, ¿qué estrategias para empoderar a las mujeres en sus decisiones de planificación familiar y de establecer compromisos y responsabilidades con sus parejas, empleadas en otras poblaciones comparables, podrían aportar a las estrategias mexicanas para lograr decisiones informadas y autónomas? Con base en la pregunta, nos propusimos definir las estrategias para empoderar a las mujeres mexicanas en sus decisiones de planificación familiar y de establecer compromisos y responsabilidades con sus parejas para lograr decisiones informadas y autónomas, incluyendo: (1) describir cómo la violencia de pareja puede afectar la decisión y apego a los métodos de planificación familiar; (2) bosquejar el contexto sociocultural de las poblaciones de las que procede la evidencia y cómo son comparables con la mexicana; (3) enunciar las estrategias que, en otros contextos poblacionales comparables, han sido efectivas para establecer compromisos y responsabilidades.

\section{Material y métodos}

Criterios de inclusión: Se realizó una revisión sistemática, incluyendo los artículos de las bases de datos PubMed, Redalyc y Scopus, filtrando los estudios publicados durante los últimos diez años. Posteriormente se confeccionó una base de datos, en la cual se codificaron y transformaron en variables las características de la publicación, los aspectos metodológicos del estudio y de los participantes, y sus principales hallazgos.

Las palabras clave identificadas y seleccionadas como criterios de inclusión fueron:

Conducta anticonceptiva. Prevención de la concepción, bloqueando la fertilidad de forma temporal o permanente (esterilización, reproductiva). Los medios comunes de anticoncepción reversible incluyen métodos de planificación natural de la familia.

Violencia doméstica. Abusos físicos deliberados, a menudo repetitivos verbales y/o de otro tipo por parte de uno o más miembros de un hogar.

Violencia de pareja. Un patrón de comportamiento agresivo y coercitivo de un individuo contra su pareja o cónyuge que puede incluir lesiones físicas, abuso psicológico, agresión sexual, aislamiento progresivo, intimidación y coerción reproductiva.

Planificación familiar. Programas o servicios de atención médica diseñados para ayudar a las personas en la planificación del tamaño de la familia. Se puede usar varios métodos de anticoncepción para control del número y el momento.

Derechos reproductivos. Los derechos reproductivos se basan en el reconocimiento del derecho básico de todas las parejas e individuos a decidir, libre y responsablemente, el número, el espacio y el tiempo de sus hijos, y a tener información y medios para hacerlo.

Criterios de exclusión: artículos de opinión, cartas al editor, protocolos en proceso, reportes de caso. 
Estrategias en relación con los derechos reproductivos y la planificación familiar - Manuel José Peón-Ponce et al.

Cuadro 1. Estructura de la base de datos y operacionalización de las variables

\begin{tabular}{|c|c|c|c|}
\hline $\begin{array}{l}\text { Variables } \\
\text { (columnas) }\end{array}$ & Dimensiones & Tipo & Rango \\
\hline $\begin{array}{l}\text { Número de } \\
\text { identificación }\end{array}$ & Números únicos & Numérica & $0-10$ \\
\hline $\begin{array}{l}\text { Base de } \\
\text { Procedencia }\end{array}$ & Nombre de la base & Categórica & PubMed, Redalyc, Scopus \\
\hline Año & Año de publicación & Numérica & $2010-2020$ \\
\hline $\begin{array}{l}\text { Apellido del primer } \\
\text { autor }\end{array}$ & Primer apellido & Categórica & Indeterminado \\
\hline País & Nombre del país & Categórica & Indeterminado \\
\hline Clave DOI & Digital Object Identifier & Categórica & Indeterminado \\
\hline Tipo de artículo & Mención a la publicación & Categórica & Original, revisión, etc. \\
\hline $\begin{array}{l}\text { Diseño } \\
\text { metodológico }\end{array}$ & De acuerdo con guías Equator & Categórica & observacional, experimental \\
\hline Cualitativo & Diseño cualitativo & Categórica & De acuerdo con Creswell \\
\hline Cuantitativo & Diseño cuantitativo & Categórica & De acuerdo con Fletcher \\
\hline Técnica descriptiva & $\begin{array}{l}\text { Analítica, sintética, inducción, } \\
\text { deducción }\end{array}$ & $\begin{array}{l}\text { Categórica } \\
\text { multinomial }\end{array}$ & $\begin{array}{l}\text { De acuerdo con Creswell Mixed } \\
\text { Methods Research }\end{array}$ \\
\hline $\begin{array}{l}\text { Unidad de } \\
\text { investigación }\end{array}$ & Unidad estudiada & Categórica & Mujer, pareja, familia \\
\hline $\begin{array}{l}\text { Variable } \\
\text { dependiente }\end{array}$ & Aquella de que deriva & Categórica & $\begin{array}{l}\text { Violencia, planificación familiar, } \\
\text { etc. }\end{array}$ \\
\hline $\begin{array}{l}\text { Variables } \\
\text { independientes }\end{array}$ & Asociadas o detonantes & Categórica & $\begin{array}{l}\text { Violencia, planificación familiar, } \\
\text { etc. }\end{array}$ \\
\hline Otras variables & Abierta & Abierta & Abierta \\
\hline Tipo de violencia & Dimensiones de la violencia & Categórica & Doméstica, sexual, íntima, etc. \\
\hline $\begin{array}{l}\text { Momento } \\
\text { reproductivo }\end{array}$ & Cronológico & Categórica & posparto, nulípara, etc. \\
\hline Grupos de edad & Edad promedio o de grupo & Abierta & discreta u ordinal \\
\hline $\begin{array}{l}\text { Contexto } \\
\text { sociocultural }\end{array}$ & Dicotómica & Menciona o no & Sí/No menciona, cuál presenta \\
\hline $\begin{array}{l}\text { Número de } \\
\text { participantes }\end{array}$ & Números específicos & numérica & Indeterminada \\
\hline $\begin{array}{l}\text { Aborda } \\
\text { problemática }\end{array}$ & Dicotómica & Menciona o no & Sí/No menciona, cuál presenta \\
\hline Aborda soluciones & Dicotómica & Menciona o no & Sí/No menciona, cuál presenta \\
\hline Aborda bioética & Dicotómica & Menciona o no & Sí/No menciona, cuál presenta \\
\hline Aborda derechos & Dicotómica & Menciona o no & Sí/No menciona, cuál presenta \\
\hline $\begin{array}{l}\text { Aborda } \\
\text { empoderamiento }\end{array}$ & Dicotómica & Menciona o no & Sí/No menciona, cuál presenta \\
\hline
\end{tabular}


Criterios de eliminación: artículos que no se encuentren con acceso en las bases de datos consultadas y aquellos que, a pesar de cumplir los criterios de inclusión, su contenido refleje un tópico deslindado con la temática propuesta.

Variables de estudio desde la bioética y desde la interdisciplina:

- Año de estudio.

- País de realización.

- Diseño y aproximación metodológica.

- Tipo de abordaje.

- Técnica descriptiva.

- Unidad de investigación (mujer, pareja, familia).

- $\quad$ Tipo(s) de violencia.

- Métodos de planificación familiar estudiados.

- Momento reproductivo de las mujeres participantes.

- Grupos de edad estudiados.

- Contexto sociocultural.

- Número de participantes.

- Abordaje bioético de la problemática.

- Abordaje de las soluciones apegado a la cultura.

- Tipo de aproximación o corriente bioética.

- Concepción filosófica/teológica de la pareja/ familia.

- Abordaje de los derechos reproductivos.

- Abordaje de la problemática desde el empoderamiento femenino.

- Consideraciones sobre la autonomía.

- Consideraciones sobre la defensa de la vida de la persona.
- Contextualización cultural de la problemática.

\section{Resultados desde la bioética}

Es común el abordaje de la bioética reproductiva desde un marco de principios rectores y, ante el problema bioético que plantea, la autonomía tiene un lugar preponderante sin duda alguna; sin embargo, reconocemos cómo la falta de jerarquización de los principios de la bioética principialista ha privilegiado desmedidamente al de autonomía ante dilemas reproductivos complejos. Si bien el concepto de "autonomía" tiene raíces filosóficas en el pensamiento de Kant y Mill, la ética basada en la autonomía tiene puntos de crítica que se deben considerar. Por ejemplo, desde la perspectiva feminista se considera un principio demasiado amplio y difícil de aplicar en lo particular, al dejar a un lado aspectos como el empoderamiento femenino para ejercer la autonomía y, sin duda, el abordaje del empoderamiento es una variable de nuestra propuesta(9-12).

El respeto a la autonomía ha mostrado un particular auge desde el marco desarrollado por Childress y Beauchamp y, aun cuando muchos autores, que no siguen explícitamente los argumentos basados en la autonomía, también utilizan el enfoque de cuatro principios y, por ello, dada la importancia de la verdadera autonomía así como la justicia y libertad en la decisión de los métodos de planificación familiar, una variable muy importante en la violencia de pareja y elección del método de planificación familiar es el abordaje de estos prin$\operatorname{cipios}(9,10)$.

Las corrientes consecuencialistas en general, y en particular la utilitarista, para la definición de variables de estudio serían estrechas, pues remitirían a pensar en el beneficio para la mayoría o a buscar la medida de maximización en la satisfacción de las preferencias de la mayoría de los interesados.

En términos prácticos, ello llevaría a pensar en números como los que reporta el INEGI, en tasas de nacimientos y de fecundidad, en la satisfacción con los anticonceptivos de emergencia y, por ello, a pesar de la creciente popularización de esta teoría propuesta por Peter Singer, no podría llevar a variables de interés. Por su parte, las corrientes 
consecuencialistas en general valoran más los fines que los medios. Ello me remitiría a pensar que el hecho de que los métodos de planificación familiar estén funcionando y que los consentimientos obstétricos estén firmados son suficientemente buenos resultados como para dedicar tiempo a analizar ¿qué tan informada estaba la paciente? La decisión de su método, ¿se ve influenciada por la pareja que la violenta? Por ello, las variables de estudio emanadas de estas corrientes no serían de nuestra elección(11-14).

Del personalismo retomaremos sus principios y la contextualización de la persona en su cultura. La defensa de la vida y dignidad humana las abordaremos desde la elección de los métodos de planificación por las mujeres que viven violencia de pareja; también desde las consideraciones sobre la persona que es la mujer y la persona del potencial producto de la gestación; lo mismo el de totalidad, pues deseamos explorar el abordaje de las diferentes dimensiones de las mujeres en la decisión de los métodos de planificación familiar en la revisión sistemática y, finalmente, el de solidaridad y subsidariedad, en el análisis del efecto en la pareja, la familia y la $\operatorname{sociedad}(15,16)$.

Desde la teología cristiana pudiera abordarse el papel de la familia, si existe entre los aspectos abordados en la revisión sistemática una aproximación a la sacralidad del matrimonio y cómo esta se afecta con la violencia de pareja. Según Albert Jonsen, "La bioética comenzó en la religión, pero la religión se ha desvanecido de la bioética"; sin embargo, en el contexto del problema bioético planteado es fundamental considerarla(17). Desde la antropología social y cultural es preciso derivar las variables el contexto geográfico y la cultura que rige en los países o regiones donde se desarrollaron los artículos incluidos en la revisión sistemática(18); mientras que, desde el Derecho, será incluida la variable de la orientación hacia los derechos reproductivos y/o derechos humanos o de la familia(19).

Desde la sociología pudiera emanar una aproximación al tipo de participantes en cada estudio revisado, si se trataba de mujeres, de parejas, de familias, para poder contextualizar los hallazgos con referencia a quienes representa(19).
Desde la metodología, en la revisión sistemática es posible identificar el tipo de diseño metodológico e identificar si el estudio aborda meramente la problemática o también sus soluciones, además de conocer la muestra participante en cada estu$\operatorname{dio}(20)$.

Desde la epidemiología y demografía, se indagarán las variables sociodemográficas de las personas a las cuales representa cada estudio, sus edades y factores epidemiológicos asociados, desde el eje temporal del año en que cada estudio fue realizado(19). A partir de la psicología puede abordarse la percepción femenina desde el momento reproductivo mismo de las mujeres, representadas en la revisión sistemática, así como el tipo de violencia de la que han sido víctimas, pues ello permitirá contextualizar los hallazgos(19).

De la ginecología y obstetricia se puede retomar variables relacionadas con el tipo de método anticonceptivo, dado el momento reproductivo de las mujeres participantes. Ello permitirá aproximarse al riesgo derivado de cada método para la mujer, dado su momento reproductivo, e incluso a la comprensión de la aceptación y apego a cada método(11).

\section{Resultados}

Diez artículos originales, entre los cuales se suma la experiencia de 90.070 personas de dos continentes y diversas etnias y culturas que participaron en los estudios. Si bien los realizados en México y en Latinoamérica abordaron extensamente la problemática de la violencia de pareja, violencia doméstica y salud reproductiva, ninguno de ellos proporcionó una propuesta o estrategia para implementar mejoras sobre la coerción sobre las mujeres que viven violencia de pareja en la adopción de un método de planificación familiar.

Los estudios revisados, en su conjunto incluyeron 90.070 personas en un periodo de diez años, entre 2010 y 2020 . El flujo de personas participantes (mujeres y sus parejas/familias) se presenta en el gráfico 1. 


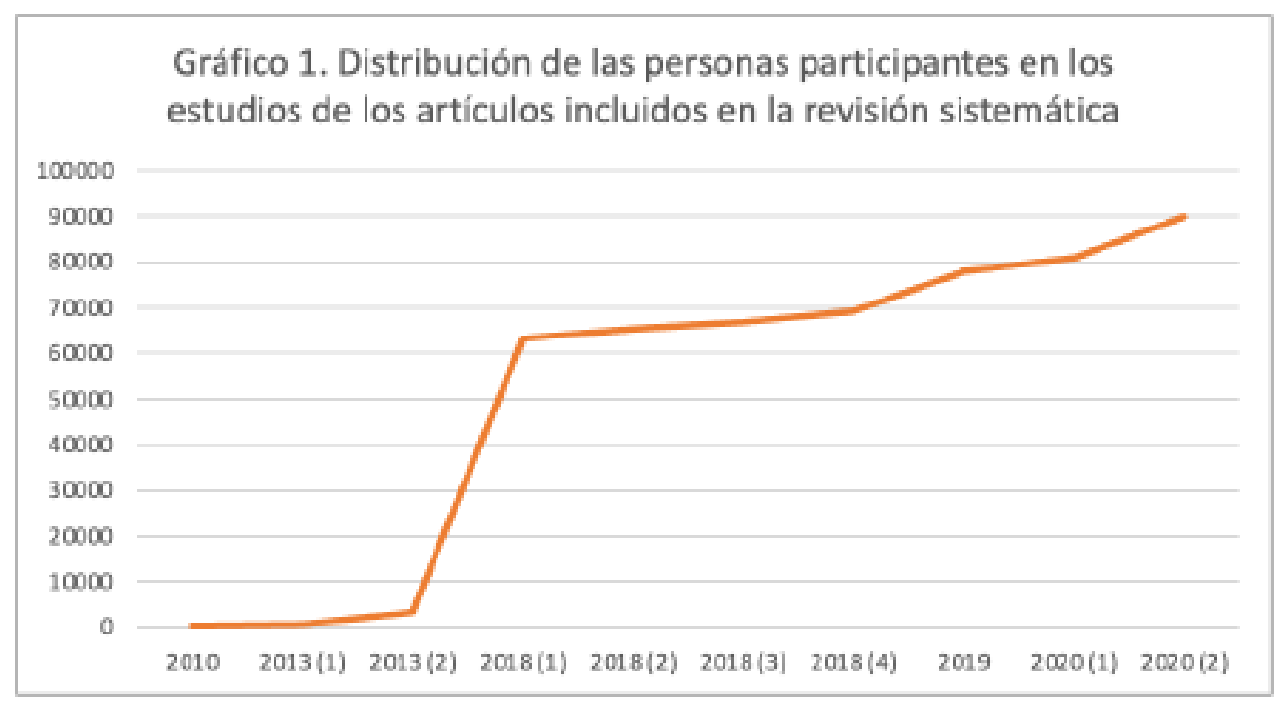

Los resultados cuantitativos obtenidos mediante la búsqueda se grafican en el cuadro 2 .

\begin{tabular}{|l|l|}
\hline Cuadro 2. Resumen cuantitativo de resultados en la investigación documental \\
\hline Variables (columnas) & Resultados cuantitativos \\
\hline Base de Procedencia & $33 \%$ de cada base \\
\hline País & 7 países de 3 continentes \\
\hline Tipo de artículo & $90 \%$ artículos originales, 10\% de revisión \\
\hline Diseño Metodológico & $80 \%$ artículos observacionales \\
\hline Cualitativo & $10 \%$ cualitativos \\
\hline Cuantitativo & $90 \%$ cuantitativos \\
\hline Técnica descriptiva & $30 \%$ analíticos, $70 \%$ descriptivos \\
\hline Unidad de investigación & $70 \%$ mujeres, 30\% parejas \\
\hline Variable dependiente & $80 \%$ método de planificación familiar \\
\hline Variables independientes & Violencia de pareja $60 \%$ \\
\hline Otras variables & Pérdidas fetales, abortos \\
\hline Tipo de violencia & $20 \%$ doméstica, 20\% sexual \\
\hline Momento reproductivo & $10 \%$ post aborto, 70\% indistinto, 20\% post evento obstétrico \\
\hline Grupos de edad & $18-45$ ańos \\
\hline Contexto sociocultural & Diversas culturas, todas patriarcales \\
\hline Número de participantes & $256-60,000$ \\
\hline Aborda problemática & $90 \%$ \\
\hline Aborda soluciones & $40 \%$ \\
\hline Aborda bioética & $10 \%$ \\
\hline Aborda derechos & $70 \%$ \\
\hline Aborda empoderamiento & $30 \%$ \\
\hline
\end{tabular}


La evidencia procedente de México, Colombia, Venezuela, Estados Unidos, India, Egipto y diversos países africanos ha documentado consistentemente que, al existir violencia de pareja (en algunos casos catalogada como "violencia doméstica" o "violencia sexual"), con cualquiera de sus manifestaciones y variedades, existe también una coerción de la libertad para elegir los métodos de planificación familiar, dificultad para el apego y coartación de los derechos reproductivos femeninos en general (22,23-25).

Las investigaciones de Kusunoki (2019), Samari (2019), Wandera (2018) y Cha (2015) identificaron que, después de que la mujer elige un método de planificación familiar, comúnmente, si este implica la participación masculina, la mujer que es violentada es más propensa a abandonarlo al poco tiempo (22,26-29).

Los estudios desarrollados en América Latina (incluyendo México) detallan cómo se presentan las interrelaciones complejas de las parejas con violencia y el camino por el cual derivan, pero aún no existe evidencia de las estrategias que pudieran mitigar esta coerción, de acuerdo con la revisión realizada para el presente escrito $(25-27,30)$.

Por su parte, según los estudios de Stevenson (2020), Memiah (2020) y Speitzer (2019), además de desentrañar los aspectos inherentes al efecto de la violencia de pareja sobre la elección de los métodos de planificación familiar, es más probable que estos métodos se suspendan al poco tiempo(28-32).

Memiah(29), incluso, resaltó el fenómeno de que, cuando se experimenta una pérdida fetal o neonatal, la aceptación de métodos de planificación familiar duraderos por parte de las mujeres es baja. Lo cual es explicado por el autor como un "intento" de, a corto plazo, reponer el hijo perdido a la pareja, aun cuando la evidencia médica indica que existe un gran riesgo reproductivo para las mujeres si se embarazan en un periodo menor a dos años (idealmente), y aún mayor si ello sucede antes de cumplirse un ańo del ultimo evento obstétrico(6).

Las soluciones a la problemática de la violencia de pareja, como modo de coerción de la libertad de elección y apego a los métodos de planificación familiar, fueron abordados únicamente, a la luz de la presente revisión, por los autores de Uganda y Centro y Sur africanos, además de los de los Estados Unidos de América. En dichos estudios se menciona la dificultad que la mujer tiene al abordar la temática de los métodos de planificación familiar a adoptar, pues se considera, de acuerdo con las culturas estudiadas, que es en cierto modo una osadía o atrevimiento que la mujer solicite al varón el uso de condón para las relaciones sexuales. Kusunoki propone que la pareja que la mujer que logra vencer esa barrera cultural y abordar el uso del condón con el varón, lo hace a través del empoderamiento y la convicción de la necesidad de atender la anticoncepción(24).

Por otro lado, Wandera sostiene que la comunicación debe estar dirigida a la pareja por parte del personal de consejería de métodos de planificación familiar, para que, una vez que la mujer decida abordar el tema en la intimidad, exista el precedente y la decisión sea más asertiva(27).

Por otro lado, Silverman asegura que el momento postevento obstétrico permite la comunicación con la mujer, y que la adopción de los métodos en esa etapa pudiera resultar más duradera, dado el momento obstétrico que vive la pareja(28).

Sin embargo, en el estudio de Memiah, con mujeres con antecedente de pérdidas fetales e infantiles, los autores permiten entrever lo subliminal y sutil que puede llegar a ser el control de la pareja masculina, a tal grado que la mujer es capaz de poner en riesgo su propia vida con tal de satisfacer los deseos reproductivos de su pareja, o de pensar que, teniendo una familia, la violencia cesaría(29).

\section{Discusión y sugerencias}

El problema bioético abordado a través de la presente revisión sistemática involucra a la población mexicana femenina, que pudiera no estar realizando una decisión verdaderamente informada y libre sobre la adopción de un método de planificación familiar, por estar viviendo violencia de pareja.

Las soluciones a un problema tan complejo han 
de operar en diferentes niveles, desde la interdisciplina y de manera constante, como se indica desde la medicina preventiva: medidas multinivel aunadas a medidas contracultura, prevención primaria y secundaria.

Las medidas contracultura, desde la medicina preventiva, son aquellas que sancionan o generan campañas contrarias a prácticas moralmente aceptadas, pero que condicionan un riesgo importante para la salud. Para ello se implementan campañas en los programas televisivos, eslóganes y mensajes clave en salud.

Las medidas multinivel involucran que no sea solo la "población blanco" a la que se dirijan las medidas propuestas, sino que, desde distintos frentes, sean tomadas las acciones sinérgicas que abonen al cumplimiento y éxito colectivo.

Por su parte, desde la prevención primaria pueden promoverse las medidas de promoción a la salud y autocuidado, las que, al ser exitosas, darán lugar al empoderamiento, gobernanza y autoeficacia en salud. La prevención primaria involucra también la capacitación e implementación de medidas para asegurar la calidad de la labor de los profesionistas de la salud.

Finalmente, la prevención secundaria permite evaluar y medir el riesgo en poblaciones especiales, como la de las mujeres que han abortado o sufrido un evento obstétrico fallido reciente, y presentan riesgos serios a su vida en caso de embarazarse nuevamente en un corto plazo.

Entre otras soluciones multinivel se puede mencionar:

(a) Un sistema de gobierno que pondere las cifras, pero que también regule adecuadamente la dispensación de píldoras anticonceptivas de emergencia, que implemente indicadores de población, natalidad y fecundidad de tipo cualitativo, aunados a aquellos de tipo cuantitativo.

(b) Aportar, mediante el ejemplo y la fuerza ciudadana, a que la sociedad mexicana sea una sociedad que no normalice la violencia a la mujer en diferentes ámbitos, que combata desde los micromachismos hasta la coerción de las decisiones libres. Diversas medidas pueden tomarse desde las aulas, en las materias de formación cívica y ética, para lograr que ese aprendizaje no sea únicamente teórico, sino que sea palpable y trascienda los muros de las aulas, para llegar a los hogares y transformarlos en nichos de equidad y justicia, pero sobre todo de paz.

(c) Evaluar cualitativamente los servicios de salud, no protocolarmente, solo en aspectos administrativos, sino ponderando el modo mediante el cual se alcanzan las metas de los centros de salud y sus profesionistas.

(d) Desarrollar campañas que permeen en las expectativas femeninas sobre cómo debe de ser la relación de pareja, para lograr que, eventualmente, los hogares violentos dejen de perpetuarse, por considerarse normales por las jóvenes mujeres que establecen sus relaciones de pareja.

(e) Empoderar a las mujeres de manera positiva y basándose en estrategias de valoración y autocuidado, para que puedan romper las barreras intangibles que les impiden abordar libremente la problemática de planificación familiar con su pareja, sin miedo, sin coerción y con la seguridad de que ambos aportarán para el apego y adherencia.

\section{Conclusiones}

La violencia ejercida en el seno de una familia o una pareja, así como la violencia sexual, tienen efecto duradero sobre la libertad de las mujeres en la elección y apego a un método de planificación familiar, cualquiera que sea su tipo y aun si no requiere de la participación por parte del varón.

Los alcances de la violencia de pareja sobre las decisiones femeninas no siempre son claramente perceptibles, de hecho, suelen ser comúnmente inadvertidas, más aún cuando la mujer toma la decisión reproductiva buscando satisfacer la voluntad de su pareja. Por ello, las consideraciones de consejería de métodos de planificación familiar no deben dirigirse únicamente a las mujeres que son violentadas, sino que el empoderamiento y autocuidado debe fomentarse aun en ausencia de antecedentes de violencia.

El empoderamiento y la autoeficacia en el cuidado 
de la salud son estrategias que paralelamente pueden abonar al diálogo de la pareja, a la decisión informada femenina e, incluso, a la participación del varón con apego y adherencia al método de planificación familiar.

Existen diferencias culturales en los contextos en los que se ha identificado que la violencia de pareja afecta la decisión libre e informada sobre los métodos de planificación familiar por parte de la mujer, pero todas las personas en las que se ha estudiado este fenómeno tienen en común que pertenecen a culturas patriarcales, en las cuales la voluntad masculina suele ponderarse más que la femenina en una relación de pareja.

Existen múltiples medidas que pueden adoptarse por los sistemas de salud, sus instituciones y su personal para mejorar la calidad de la consejería en métodos de planificación familiar y el consentimiento informado para fines obstétricos, pero es importante que el personal esté capacitado, lo cual es una función a la cual los bioeticistas pueden aportar desde los comités hospitalarios de bioética y de ética en investigación de las instituciones de salud y las universidades.

\section{Recomendaciones y sugerencias}

La problemática compleja que subyace a la ausencia de una verdadera autonomía y justicia, como principios rectores, en la elección de un método de planificación familiar por parte de la mujer violentada no es banal, los bioeticistas, desde los comités de bioética podemos y debemos generar espacios para implementar mejoras en los procesos de consentimiento informado, desarrollar estrategias para asegurar que el personal de salud sea consciente de la importancia del cuidado del proceso.

El personal de salud puede capacitarse y tener conciencia de cuán importante es la persona, su dignidad, el cuidado de su vida y el beneficio que puede otorgársele con un buen proceso de orientación e indicación de métodos de planificación familiar. Si el personal de salud pudiera aspirar a comprender un poco más el contexto de la paciente, su entorno cultural, y preguntarse qué tan bien informada esta la paciente, y si la decisión de su método se ve influenciada por la pareja, po- dríamos lograr mejoras no solo en el proceso, sino también en el apego.

De acuerdo con la revisión, para el personal de salud es prácticamente imposible identificar si cada paciente que llega para consejería de métodos de planificación familiar es o no una persona violentada por su pareja, o si su libertad de decisión se encuentra limitada por los deseos de cumplir los deseos del varón, pero lo que sí puede hacerse, en todos los casos, es tomar en cuenta la posibilidad de que así sea y fomentar en cada consejería el empoderamiento del cuidado de la salud reproductiva como un derecho de toda mujer, $y$, mediante la consejería, identificar y fomentar aquellas estrategias que pudieran facilitar el diálogo de pareja.

Adicionalmente, después de un evento obstétrico adverso o no adverso, para las mujeres puede privilegiarse un buen proceso de consejería y consentimiento obstétrico informado, que proteja la vida humana materna y prevenga del riesgo inherente a un nuevo embarazo cercano a la madre con comorbilidades. También es menester favorecer los métodos que no impliquen meramente impedir la implantación del huevo ya fecundado y, con ello, es importante informar de manera completa sobre el funcionamiento de las píldoras anticonceptivas de emergencia. Esto puede ser desarrollado por el personal de salud, como es costumbre, pero, como bioeticistas, podemos implementar capacitaciones continuas en nuestras instituciones, con la finalidad de abordar estos tópicos con el personal de salud.

El acompańamiento psicológico de las parejas, familias y mujeres que viven en violencia es realmente importante; sin duda, en el diseño de capacitaciones y sesiones informativas para parejas en búsqueda de opciones de métodos de planificación familiar es fundamental la interdisciplina.

Los cambios sociales que abonen a una sociedad más justa, informada y pacífica son menester de todos los ciudadanos, y a nosotros, como bioeticistas, nos toca ser motores que inicien y mantengan las tendencias hacia la sociedad que queremos. Para ello es importante nuestro involucramiento, nuestra participación en diferentes sectores de la sociedad, en nuestras instituciones, y en todo momento debemos modelar con nuestro ejemplo. 


\section{Referencias}

1. Instituto Nacional de Estadística y Geografía. Natalidad y fecundidad. México [En línea]. Disponible en http://www. beta.inegi.org.mx/temas/natalidad/

2. Consejo Nacional de Población. Indicadores demográficos básicos 1990-2030. Disponible en http://www.conapo.gob. $\mathrm{mx} /$ index.php?option=com_content \&view=article\&id=125\&Itemid=193.

3. Secretaria de Salud. Norma Oficial Mexicana, NOM 005-SSA2-1993, De los Servicios de Planificación Familiar. México. Disponible en http://www.salud.gob.mx/unidades/cdi/nom/005ssa23.html

4. Cetina-Sauri G, Huchim-Lara O, Alvarez-Baeza A, Inurreta-Díaz M, Puga-Matu H, Aguilar-Vargas E, Méndez-Domínguez N. Undergraduate medical students. Simulation-based activity to conduct the informed consent process for health research studies. Educación Médica 2018; 21(2): 106-111.

5. Inurreta-Díaz M, Morales-Gual YM, Aguilar-Vargas E, Álvarez-Baeza A, Magriñá-Lizama JS, Cetina-Sauri G, MéndezDomínguez N. Family-Planning counselling simulation for medical students: An exploratory educational intervention. Educación Médica 2020; 22(54): 271-276.

6. Abraham ZG, Heidy OR, Julie SK, Carla PA, Raymundo PR. Periodo intergenésico: Revisión de la literatura. Revista chilena de obstetricia y ginecología 2018; 83(1)

7. Mandujano JC, Reyes de la Cruz C, Sarmiento A, Murillo AL, Osorio DR. Conocimiento y uso de la pildora de emergencia en jóvenes universitarias de Tabasco. Universidad Juárez Autónoma de Tabasco; 2018. DOI: 10.19136/hs.a17n3.2161

8. Villegas JS, Santiago ML, Quinteros RS. Riesgos y daños a la salud de las adolescentes por el uso de la pastilla de emergencia. Revista Electrónica de Investigación en Enfermería FESI-UNAM 2012; 1(1). Disponible en: http://journals. iztacala.unam.mx/index.php/cuidarte/article/viewArticle/197

9. Jonsen, A. R. A History of Religion and Bioethics, in Handbook of Bioethics and Religion, eds. David E. Guinn. Oxford: Oxford University Press; 2006: 23-35.

10. O’Neill, O. Autonomy and Trust in Bioethics. Cambridge: Cambridge University Press; 2002.

11. Mackenzie C, Stoljar N, (eds.). Relational Autonomy: Feminist Perspectives on Autonomy, Agency and the Social Self. Oxford: Oxford University Press; 2000.

12. Messer N. Bioethics and Practical Theology: The Example of Reproductive Medicine. International Journal of Practical Theology 2017; 21(2): 291-314.

13. Beauchamp T, Childress JF. Principles of Biomedical Ethics. 7th ed. New York: Oxford University Press; 2012.

14. Singer P. Practical ethics. Cambridge University Press; 2011.

15. Díaz EF. La cuestión del género, ¿̇un aporte a la comprensión de la mujer? Una reflexión desde la bioética personalista. Revista del Cuerpo Médico Hospital Nacional Almanzor Aguinaga Asenjo 2013; 6(4): 47-56.

16. Velasco JM. ¿Qué es la bioética personalista? Un análisis de su especificidad y de sus fundamentos teóricos. Cuadernos de bioética 2013; 24(1): 17-30.

17. Jonsen AR. A history of religion and bioethics. In Guinn D. E. (ed.). Handbook of Bioethics and Religion. Oxford University Press; 2006.

18. Marshall PA. Anthropology and bioethics. Medical Anthropology Quarterly 1992; 6(1): 49-73.

19. Post SG. Encyclopedia of Bioethics, 5 Volume. Set. Gale; 2004.

20. Davies R, Ives J, Dunn M. A systematic review of empirical bioethics methodologies. BMC Medical Ethics 2015; 16(1): 15.

21. McKneally MF, Singer PA. Bioethics for clinicians: 25. Teaching bioethics in the clinical setting. Cmaj 2001; 164(8): 1163-1167.

22. Cha, S, Chapman, DA, Wan, W, Burton, CW, Masho SW. Intimate partner violence and postpartum contraceptive use: The role of race/ethnicity and prenatal birth control counseling. Contraception 2015; 92(3): 268-275.

23. Forrest, W, Arunachalam, D, Navaneetham K. Intimate partner violence and contraceptive use in India: The moderating influence of conflicting fertility preferences and contraceptive intentions. Journal of Biosocial Science 2018; 50(2): 212-226.

24. Kusunoki Y, Barber JS, Gatny, HH, Melendez R. Physical intimate partner violence and contraceptive behaviors among young women. Journal of Women's Health 2018; 27(8), 1016-1025.

25. López Rosales F, Moral de la Rubia J, Díaz Loving R, Cienfuegos Martínez YI. Violencia en la pareja. Un análisis desde una perspectiva ecológica. CIENCIA ergo-sum, Revista Cientifica Multidisciplinaria de Prospectiva 2013; 20(1), 6-16. Disponible en https://www.redalyc.org/articulo.oa?id=104/10425466009 
26. Samari G. Women's empowerment and short-and long-acting contraceptive method use in Egypt. Culture, health \& sexuality 2018; 20(4), 458-473.

27. Wandera SO, Kwagala B, Odimegwu C. Intimate partner violence and current modern contraceptive use among married women in Uganda: a cross-sectional study. The Pan African medical journal 2018; 30.

28. Silverman JG, Boyce S.C, Dehingia N, Rao N, Chandurkar D, Nanda P, ... Raj A. Reproductive coercion in Uttar Pradesh, India: Prevalence and associations with partner violence and reproductive health. SSM-Population Health 2019; 9, 100484.

29. Memiah P, Bond T, Opanga Y, Kingori C, Cook C, Mwangi M, ... Owuor K. Neonatal, infant, and child mortality among women exposed to intimate partner violence in East Africa: a multi-country analysis. BMC Women's Health. 2020; 20(1), 10.

30. Ocampo Otálvaro LE, Amar Amar JJ. Violencia en la pareja, las caras del fenómeno. Salud Uninorte 2011; 27(1), 108-123.

31. Speizer IS, Pettifor A, Cummings S, MacPhail C, Kleinschmidt I, Rees HV. Sexual violence and reproductive health outcomes among South African female youths: a contextual analysis. American Journal of Public Health 2010; 99(S2), S425-S431.

32. Rodríguez F, Córdova L. Violencia en la pareja: manifestaciones concretas y factores asociados. Espacio Abierto 2009; $18(2), 323-338$.

Recibido: 25 de mayo de 2021

Aceptado: 19 de julio de 2021 